\section{LEOPOLDO CALVO-SOTELO LA TRANSICIÓN EXTERIOR: PRIORIDAD EUROPEA}

\section{Y \\ LA}

\author{
Pablo Pérez López \\ Universidad de Navarra \\ paperezlo@unav.es \\ Jorge Lafuente del Cano \\ Universidad de Valladolid
}

Cómo citar este artículo/Citation: Pérez López, P. y Lafuente del Cano, J. (2014). "Leopoldo Calvo-Sotelo y la Transición exterior: la prioridad europea". Arbor, 190 (769): a169. doi: http://dx.doi.org/10.3989/arbor.2014.769n5008

Recibido: 3 marzo 2014. Aceptado: 5 junio 2014.

RESUMEN: Leopoldo Calvo-Sotelo desempeñó importantes responsabilidades ejecutivas en los gobiernos de la Transición, incluida la presidencia del Gobierno. Su gestión estuvo intensamente vinculada a las relaciones exteriores, dentro del proyecto que él mismo denominó «transición exterior». El artículo, basado en buena medida en documentación conservada en su archivo personal, analiza cuáles fueron sus principales ideas y en qué medida su concepto de Europa desempeñó un papel relevante en la formulación y ejecución de su política. Analiza esos hechos en relación con otros dirigentes del momento, especialmente Adolfo Suárez y Felipe González. Las conclusiones permiten conocer en qué consistió el europeísmo del principal protagonista de estos hechos.

PALABRAS CLAVE: España; Transición a la democracia; relaciones internacionales; Europa; Leopoldo Calvo-Sotelo; OTAN.

\section{LEOPOLDO CALVO-SOTELO AND THE FOREIGN TRANSITION: THE PRIORITY OF EUROPE}

Copyright: (C) 2014 CSIC. Este es un artículo de acceso abierto distribuido bajo los términos de la licencia Creative Commons Attribution-Non Commercial (by-nc) Spain 3.0.

ABSTRACT: Leopoldo Calvo-Sotelo played an important role in Spain's transition to democracy, serving as minister and later prime minister. His political career was closely linked to Foreign Affairs and to the process of the "Foreign Transition" as he liked to call it. This paper, largely based upon his personal archives, analyses his thought, and how his idea of Europe played an important role in the formulation and execution of his policy. Calvo-Sotelo's ideas and achievements are compared with those of other political leaders of the time, such as Adolfo Suárez and Felipe González. The conclusions show the nature and characteristics of Leopoldo CalvoSotelo's pro-European ideas.

KEYWORDS: Spain; Transition to democracy; International Relations; Europe; Leopoldo Calvo-Sotelo; NATO. 


\section{INTRODUCCIÓN}

La Transición a la democracia vivida tras el régimen de Franco tuvo una importante dimensión exterior que ha concitado interés creciente por parte de los especialistas. Estamos lejos, sin embargo, de tener un conocimiento de esa dimensión internacional parangonable al que tenemos de la nacional. El presente artículo pretende una aportación a esa historia centrada en quien fuera uno de sus principales protagonistas, Leopoldo Calvo-Sotelo. El conocimiento de su punto de vista, de su programa, de sus logros y de sus fracasos arroja luz sobre el proceso, y tiene una ventaja añadida: implica reflexionar sobre las ideas -al menos las más importantes- de quienes le precedieron y sucedieron en la presidencia del Gobierno, Adolfo Suárez y Felipe González. Ese análisis comparativo permite enriquecer nuestro conocimiento del relieve de ese paisaje internacional.

El punto de vista de Calvo-Sotelo nacía de su concepto de política internacional y de cómo debe abordarse. Su idea tenía mucho de planteamiento estratégico y se enraizaba en la historia como lugar de definición de España, también en el ámbito internacional. La interpretación que Calvo-Sotelo hizo de esa historia explica sus proyectos y decisiones. El contraste entre estas y las de otros destacados políticos, unos centristas como él, otros socialistas, pone de manifiesto la importancia de la comprensión personal de la historia en la toma de decisiones políticas.

\section{UN EUROPEÍSMO ARRAIGADO: INTELECTUAL, PROFESIONAL Y POLÍTICO}

La bibliotecaria que trabajó más de diez años en la ordenación de los casi once mil volúmenes que formaban la biblioteca personal de Leopoldo Calvo-Sotelo, ha evocado así lo que esta traslucía de su creador:

«tiene las características propias de un humanista, interesado por múltiples ramas del saber, comprometido con la historia de su país, y con una profunda vocación europea» (Fernández Palomeque, 2010, p. 85).

En efecto, el interés por Europa y lo europeo destaca como un rasgo sobresaliente en el perfil intelectual y político del ex presidente de Gobierno, y lo hace desde una edad temprana. Ingeniero de Caminos de formación, en sus tiempos de estudiante demostró un interés por las cuestiones de pensamiento que le llevaron participar en actividades religiosas y culturales de la Asociación Católica Nacional de Propagandistas y a frecuentar las conferencias de Xavier Zubiri y los cursos de José Ortega y Gasset en el Instituto de Hu- manidades de Madrid (Calvo-Sotelo Ibáñez-Martín, 2010, p. 51). La influencia de Ortega, al que leyó, releyó y reconoció como el autor más influyente en él, fue el marco de su europeísmo, incrementado con la lectura de los autores de la Generación del 98, en especial Unamuno, en los que Europa - enfocada de un modo u otro, con admiración o frontal rechazo- ocupaba un papel principal.

La lectura de Ortega, por otro lado, significó la adopción de ciertos giros, usos y metáforas que el ex presidente incluía en su repertorio literario. Por ejemplo, el 29 de enero de 1993 pronunció una conferencia en Valladolid titulada «La dualidad en la construcción de Europa». Al comienzo de su intervención rememoró su primera intervención pública, casi medio siglo antes, precisamente en la capital castellana. Ambas intervenciones tenían un ingrediente común:

"Vuelvo ahora a Valladolid para hablar de Europa, continuando en el tiempo aquella primera intervención (...). Acabé hace ahora cuarenta y cinco años con una cita de Ortega y Gasset, empiezo hoy con otra, también de Europa. En el prólogo para franceses de "La rebelión de las masas", escrito durante la guerra civil en su exilio holandés, Ortega se atrevía a anunciar "una posible, una probable unidad estatal de Europa" (...).Ortega adivinó con lucidez cuáles serían los motores y los problemas de la unión política de Europa. Y lanzó el reto político de inventar una estructura capaz de hacer uno lo plural (ALCS, caja 41, exp. 9).

No encontraba programa político en el filósofo, sino un esquema intelectual, un marco de desarrollo en el que Europa («muchas abejas y un solo vuelo») aparecía como un destacado ideal («ese extraño artefacto estatal») al que España no podía renunciar (Powell, 2010, p. 104).

Este europeísmo intelectual, que se reforzó y creció cuando comenzó su ejercicio profesional, le condujo a crear una biblioteca «casi más europea que española». Casi un tercio de su libros estaban en lenguas foráneas de las que era lector: 119 libros en alemán, 2.172 en francés, 1.040 en inglés, 128 en italiano y 63 en portugués (Calvo-Sotelo IbáñezMartín, 2010, p. 52). En el catálogo se pueden contar unos 500 libros de tema europeo (un 4,5\% del total), y no pocos de historia de países europeos: entre los más numerosos alrededor de 60 sobre Italia, 80 sobre el Reino Unido, cantidad muy parecida sobre Alemania, y 280 sobre Francia (Elaboración propia a partir del catálogo de la biblioteca de Leopoldo Calvo-Sotelo, ALCS). 
Una de las razones de esta circunstancia fueron los frecuentes viajes que debió realizar por motivos profesionales por diversos países europeos, especialmente Alemania, Francia, Italia, Holanda, Bélgica, Portugal y el Reino Unido. En efecto, Leopoldo Calvo-Sotelo comenzó su trabajo en la empresa privada. El servicio de Estudios Industriales del Banco Urquijo fue su primer destino, y dentro del grupo industrial de esa entidad estuvieron sus siguientes puestos: en Perlofil, una empresa del nailon, entre 1953 y 1963, y en Unión de Explosivos Riotinto entre 1963 y 1975. Su labor en esas empresas le obligó a salir con frecuencia de España, sobre todo a Europa: «mucho a París y Bruselas, menos a Inglaterra» (Powell, 2010, p. 99), mucho también a Holanda y Alemania, «A Alemania he ido una o dos veces al mes durante quince años» (Powell, 2010, p. 102), y no pocas veces a Portugal, también por razones familiares, ya que sus suegros residieron allí de 1958 a 1967, mientras José Ibáñez Martín fue embajador en ese país.

Esa necesidad viajera, no obstante, no se hubiera convertido en oportunidad europeísta sin su curiosidad intelectual y su temprano interés político por la construcción de un proyecto europeo común. Nos consta por su propio testimonio y por el de amigos de sus tiempos de estudiante como el embajador Juan Durán-Loriga:

«(...) de aquel círculo [de la ACNdP a finales de los cuarenta] surgió algo que fue muy importante para nosotros: el europeísmo. De allí es el origen de la Asociación Española de Cooperación Europea. Un grupo de amigos, entre los que figuraban Leopoldo Calvo-Sotelo y Gonzalo Fernández de la Mora, intentamos fundar la primera asociación europeísta. Y digo intentamos porque esa sociedad, que iba a denominarse ACIES de Europa (...) no fue autorizada por la Dirección General de Seguridad, por considerar que podía tener un carácter peligroso. (...) Creíamos que la solución pacífica de los problemas de España tenían que venir a través de la vinculación de España con Europa. Esto era algo muy profundamente sentido por Leopoldo Calvo-Sotelo» (Gómez Santos, 1982, p. 96. Entrevista con Juan Durán-Loriga, 5/10/2011).

Nada extraño, pues, que uno de los primeros libros que nos consta como adquirido por él en el extranjero, en Londres concretamente, fuera una obra sobre Europa (Calvo-Sotelo Ibáñez-Martín, 2010, p. 67). En su estudio sobre la biblioteca de su padre, Pedro Calvo-Sotelo fija de manera particular su mirada en la colección Que sais-je? de Presses Universitaires de France, la colección más importante de la biblioteca por extensión y uso, que puede tomarse como ejemplo representativo del conjunto de sus libros. En ella, sin que pretendamos una enumeración exhaustiva, encontramos títulos como Les transports en Europe, 1963; L'Union Politique de l'Europe, 1973; Le Marché Commun, 1977; Les relations extérieures de la CEE, 1980; L'Union Européenne, 1994; y La constitution européenne, 2004 (Calvo-Sotelo Ibáñez-Martín, 2010, p. 61). Gracias al análisis cronológico de adquisiciones realizado por Fernández Palomeque sabemos que ese interés por los asuntos europeos le acompañó también después de abandonar su vida pública, ya que en 1992 la colección de libros sobre la Comunidad Económica Europea aumentaba cada semana (Fernández Palomeque, 2010, p. 94).

Así pues, Leopoldo Calvo-Sotelo encontró razones culturales, profesionales y políticas para interesarse por los asuntos europeos en los que veía una veta de soluciones por ser el contexto en el que se desarrollaba la vida española. De ahí que uno de sus afanes políticos fuera trasladar esa idea a la realidad política de su país. Parafraseando a Adolfo Suárez, buscó elevar a categoría política de normal lo que a nivel de calle era simplemente normal: el carácter europeo de la vida española.

\section{UNA TRAYECTORIA POLÍTICA VINCULADA A LAS RELACIONES EXTERIORES}

Leopoldo Calvo-Sotelo, monárquico y europeísta, vio cumplidos en sus años de madurez dos de sus ilusiones juveniles cuando se convirtió en actor principal de los acontecimientos políticos tras la muerte de Franco: la Transición. Fue diputado, ministro, vicepresidente y presidente del Gobierno de España, y también diputado en el Parlamento Europeo y miembro de la Asamblea Parlamentaria del Consejo de Europa.

Su carrera política de primer nivel se inició en el primer Gobierno de la Monarquía, presidido por Carlos Arias Navarro (diciembre de 1975-julio de 1976), como ministro de Comercio, ocupación que conocía bien tras veinticinco años dedicado a la empresa privada. Inició entonces una vinculación con la política exterior que mantuvo como una constante en sus años de actividad pública (Discurso del Ministro de Comercio en Barcelona, AGA, Ministerio de Comercio, no 12847, exp. 128). Efectivamente, el por entonces ministro de Asuntos Exteriores, José María de Areilza, le encargó un primer contacto con la Comunidad Europea, a la vez que él iniciaba su gira por las capitales europeas presentando el cambio que en España tendría lugar en los próximos años (Gómez Santos, 1982, pp. 163-164). Calvo-Sotelo 
se desplazó a Bruselas, entre el 25 y el 28 de abril de 1976, para reunirse con el presidente y el vicepresidente de la Comisión, a los que les trasladó el mensaje de que tras la muerte de Franco se había comenzado una nueva etapa, en la que España estaba decidida a culminar sus proceso democratizador e, inmediatamente después, a solicitar la adhesión en la CEE (Nota sin firma de la Dirección General de Relaciones Económicas Internacionales para el Ministro de Asuntos Exteriores: «Razones que aconsejan la continuación de las negociaciones España-CEE», AMAE, caja 9, 15573). Quedaba así patente la dualidad comunitaria, con su parte política y su sección económica, pilotada por el titular de Comercio.

En su etapa como ministro de Obras Públicas (julio de 1976 - abril 1977), mantuvo su preocupación por la cuestión europea, como pone de manifiesto su correspondencia con Alberto Ullastres, embajador cerca de las Comunidades Europeas (ALCS, Relaciones con la CEE, caja 51, exp. 5). Después, como portavoz del Grupo Parlamentario de UCD -ya en el Gobierno de Suárez, tras las primeras elecciones democráticas- asumió la tarea de emprender la negociación para la adhesión de España al Mercado Común, tras la petición de adhesión llevada a cabo por el ministro de Asuntos Exteriores, Marcelino Oreja. A pesar de la polémica sobre el mejor modo de llevar a cabo la negociación, Suárez se decidió por la creación de un Ministerio adjunto para las Relaciones con las Comunidades que, finalmente, ofreció a Calvo-Sotelo, buen conocedor de la realidad europea, políglota y viajero.

Calvo-Sotelo tuvo que crear la estructura del nuevo ministerio desde cero. Se rodeó de un pequeño y eficaz equipo de confianza, estableciendo las bases de la negociación, coordinando las etapas básicas con la Misión de España ante la CEE y explicando dentro y fuera de nuestro país por qué España podía y debía formar parte de la institución europea ${ }^{1}$.Tras más de dos años en el cargo (febrero de 1978 - septiembre de 1980) dejó la negociación en un avanzado estado de desarrollo frenado por la brusca interrupción giscardiana. Fue esta una etapa especialmente gratificante para su protagonista, en la que pudo combinar su pasión por Europa con la precisión técnica y la dureza diplomática que requería una larga negociación.

«Mi larga lucha en la arena política (tres Ministerios, una Vicepresidencia y la Moncloa) - dejó escrito- responde a ese cuadro pesimista del oficio público, salvo en un paréntesis soleado y casi placentero: los tres años de negociación con el Mercado Común, los tres años en los que fui Ministro para las
Relaciones con las Comunidades. Probablemente esa excepción se deba al carácter singular que tuvo aquella función negociadora dentro de la Administración Pública» (Calvo-Sotelo, 1990, p. 143).

En el último Gobierno de Adolfo Suárez (septiembre de 1980 - febrero de 1981), Calvo-Sotelo fue nombrado vicepresidente segundo del Gobierno y ministro de Economía. Alejado de la responsabilidad directa de la adhesión, siguió supervisando la negociación en la que el apartado económico adquiría una importancia cada vez más destacada.

Tras la dimisión del presidente del Gobierno en enero de 1981, Calvo-Sotelo fue elegido como sucesor en la Moncloa. Entre las prioridades de su Gobierno, plasmadas en su discurso de investidura, estaba la política exterior, con la mención expresa del deseo de ingresar en el Mercado Común y en la Alianza Atlántica (Calvo-Sotelo, 1982, pp. 19-21).

Desde la Presidencia del Gobierno, reformó la estructura negociadora, creando una Secretaría de Estado para las Relaciones con Europa, cuyo primer titular fue el hasta entonces embajador de la Misión de España ante la CEE, Raimundo Bassols, bajo la supervisión del ministro de Exteriores, José Pedro Pérez-Llorca. El presidente siguió atento al proceso negociador, presidiendo las Comisiones de Asuntos Exteriores, así como en sus viajes por las capitales europeas, especialmente París. Cuando UCD dejó el Gobierno la negociación estaba encauzada (Bassols, 1995, p. 276).

Desde Moncloa, además, Calvo-Sotelo se preocupó de la otra clave de la política exterior: la entrada de España en la OTAN. Cumpliendo su deseo de investidura, el Gobierno dio los pasos necesarios para incorporar a nuestro país a la Alianza Atlántica, a pesar del rechazo de la oposición. Se situó así a España en uno de los dos bloques en los que se dividía entonces el mundo. España se convirtió en 1982 en el 16 miembro de la Organización Atlántica.

Finalizada su etapa en la Presidencia, Calvo-Sotelo fue miembro de la Asamblea Parlamentaria de Europa en 1983, y diputado del Parlamento Europeo en 19861987. Fue quizá el final europeísta de una carrera política vinculada a la política exterior de España y, de manera especial, a Europa. Pero no fue ese su último quehacer relacionado con el proyecto europeo.

«Al cesante - escribió- le llueven peticiones de conferencias, artículos, prólogos, mesas redondas y demás andanzas por el estilo, que son tareas arduas y mal pagadas» (Calvo-Sotelo Ibáñez-Martín, 2010, p. 30). 
Leopoldo Calvo-Sotelo dedicó un número creciente de esas actividades a analizar ya con cierta perspectiva la política exterior española en los años de Transición y los inmediatamente posteriores, y el siempre incierto caminar de la Unión Europea de la que España era ya, por fin, miembro de pleno derecho. Desde su retiro político, en 1987, tenemos documentadas más de 45 intervenciones públicas suyas con Europa como telón de fondo (Elaboración propia a partir del catálogo del archivo de Leopoldo Calvo-Sotelo, ALCS).

\section{LA POLÍTICA EXTERIOR COMO TOMA DE POSICIÓN}

Leopoldo Calvo-Sotelo sintetizó alguna vez su idea de las relaciones internacionales con una metáfora muy clarificadora de su punto de vista:

«Hay una posición ante los problemas internacionales que parece madura, muy reflexiva que es la de los que dicen que el mundo internacional es un mundo muy duro, que hay que practicar el toma y daca, que hay que obtener cosas a cambio. Bien, esa es una posición elemental y un poco provinciana, un poco de feria o de mercado; porque la realidad no es así. Más bien lo que se juega en el mundo internacional son posiciones, lo que hay es que estar en posiciones. Ocupar posiciones que son o que sean dominantes, que permitan, a lo largo de mucho tiempo, obtener ventajas permanentes. Yo fui en tiempo aficionado a jugar al ajedrez; no sé si aquí hay algún aficionado: los que lo sean me entenderán si les digo que el jugador de ajedrez que va a tomar piezas del adversario no siempre gana la partida; gana el que tiene una posición buena y de esa posición buena deduce luego el triunfo final» (Conferencia «La Transición política exterior», Barcelona, 18 de diciembre de 1985, ALCS, caja 35, exp. 3).

Así pues el hombre de empresa, el ex ministro de Comercio, no entiende las relaciones como un mercadeo de intereses. Más bien las concibe como los estrategas miran los campos de batalla, como un problema que en su radicalidad es de posiciones. O con una imagen más pacífica, como ven los pensadores los conocimientos, como lugares seguros por conocidos o inseguros por ignotos. Desde los primeros cabe conquistar los segundos, pero no al revés.

En su discurso de entrada en la Real Academia de Ciencias Morales y Políticas el 16 de noviembre de 2005, titulado «Sobre la Transición exterior», sintetizó su idea de la historia de las relaciones exteriores españolas con su habitual estilo sentencioso, elegante y frecuentemente irónico. Cabe resumir su idea central diciendo que España abdicó de desempeñar un papel en la escena internacional desde tiempos de Carlos III y no lo recuperó hasta la Transición, en concreto hasta su entrada en la OTAN en 1982 y en las Comunidades Europeas en 1986. Para casi todos los lectores la mención de Carlos III plantea un interrogante, más todavía cuando en este apunte cronológico Calvo-Sotelo se distancia del criterio de Ortega y Gasset, que ponía en 1580 el límite de la influencia española en la escena mundial. Afortunadamente, tenemos una respuesta facilitada por el autor de la proposición:

«(...) todavía en 1774, cuando la primera crisis de las Malvinas, bastó la voz del Embajador de España en Londres - que tenía detrás una escuadra no invencible, ya con las ansias de su muerte en Trafalgar - bastó una voz respaldada solo por la historia para obtener la evacuación de los ingleses» (Calvo-Sotelo, 2005, p. 27).

De ahí en adelante España perdió la voz en los asuntos mundiales y enfermó de un mal que tuvo por síntoma lo que nuestro autor denomina «una antología de neutralidades». La más grave habría sido la de la Gran Guerra de 1914, cuando el país llegó a encontrarse, en palabras de José Echegaray «fanático de la neutralidad». Según Calvo-Sotelo, la opinión española llegó a ese estado

«ajena a las cuestiones exteriores, cuando tuvo que enfrentarse a una cuestión exterior de primerísima magnitud sin herramientas para analizarla y sin voluntad para intervenir en ella» (Calvo-Sotelo, 2005, p. 37).

Más tarde, la guerra civil habría llevado la dialéctica de las dos Españas a las relaciones exteriores y, terminada la contienda, habría vuelto la pasión por la neutralidad. La acción exterior inicial de Franco consistió en una limitada política en Iberoamérica y los países árabes, más bien retóricas. En 1953, no obstante, se llegó en palabras de Leopoldo Calvo-Sotelo, a un

«punto de inflexión decisivo de la política exterior española después del Desastre: la decisión de tomar partido, de elegir puerto, de anclar a España en el mundo occidental. Esa orientación occidental, incoada apenas, y la Monarquía sólidamente asentada son las dos grandes herencias que nos dejó el régimen anterior. Quienes estuvimos en los Gobiernos de la Transición dimos, apoyando a la Corona y asegurando el anclaje occidental de España, los primeros pasos de una nueva época» (Calvo-Sotelo, 2005, p. 43).

No hay duda de que hay una neta continuidad entre el pensamiento del joven estudiante monárquico europeísta y el ex presidente que mira la historia española desde sus años de plenitud. En esa interpre- 
tación está en buena medida la clave de su política exterior y de su interés por la cuestión europea.

El apartamiento de nuestro país de los intereses mundiales le parecía una incongruencia si no una traición a lo que era si no la esencia al menos la herencia y por tanto parte importante de la identidad nacional española. España era parte de Europa, de Occidente, y si no actuaba conforme a esos parámetros, erraba (Calvo-Sotelo, 1982, p. 86).

La tremenda división europea de las guerras mundiales había sido ajena a nosotros, según Calvo-Sotelo indebidamente. Cuando esas divisiones se habían soldado y cuando Europa había dejado de ser cabeza de los asuntos mundiales, con mayor razón España debía sentirse llamada a reintegrarse en el concierto europeo y occidental, porque ese era el lugar que le correspondía si no quería generar nuevas incoherencias que nos acabaran arrojando en penosas y estériles disputas internas. Esa parecía ser la lección que extraía de la "antología de neutralidades» que, según él, había poblado para desgracia de los españoles dos siglos de su historia. No tenía sentido aislar a España, porque España no era un verso suelto sino parte del poema europeo.

\section{ACUERDO Y DESACUERDO CON ADOLFO SUÁREZ}

Evocado su pensamiento sobre el asunto, detengámonos ahora en aspectos más prácticos. Uno de los más interesantes es el grado de coincidencia o discrepancia entre Adolfo Suárez y Leopoldo CalvoSotelo en estos asuntos. El estudio de Antonio Marquina sobre la política exterior de la UCD parece indicar un predominio personal de Adolfo Suárez en ciertas cuestiones por encima de sus ministros y aún del programa político de la UCD (Marquina, 1996, p. 187). Martínez Lillo precisa este dato cuando señala la influencia de los asesores de Suárez en la Moncloa, los Ilamados «fontaneros», en la orientación «tercermundista» de la política exterior española y la búsqueda de una «tercera vía» en el mundo bipolar de la guerra fría (Martínez Lillo, 1996, pp. 166-167).

Volveremos sobre el presidente y su equipo de asesores, pero antes detengámonos a considerar el equipo responsable de exteriores en los gobiernos Suárez. Marcelino Oreja Aguirre fue su ministro de Asuntos Exteriores casi todo el tiempo, desde julio de 1976 hasta septiembre de 1980, y lo fue más por consejo de la Corona que por elección de Adolfo Suárez (Fuentes, 2001, pp. 152-153). Oreja había sido uno de los políticos que recelaron del nombramiento de Suárez, convencido de que el papel le venía grande al abulense. Hasta llegó a pensar en no aceptar el nombramiento en un Gobierno al que auguraba poco futuro (Oreja Aguirre, 2011, pp. 141-143). Impulsado por el rey Juan Carlos modificó su postura, entró en el Gobierno, y terminó por cambiar su juicio sobre Suárez, pero sin que eso evitara desacuerdos y cierta distancia en la relación con el presidente, que pudo hacer valer una postura más firme en estos asuntos cuando el núcleo de políticos suaristas fue creciendo en el seno del Gobierno. Una de las manifestaciones de esa circunstancia fue la creación del nuevo Ministerio para las Relaciones con las Comunidades, que sustrajo esa importante tarea al de Asuntos Exteriores: a pesar de que Oreja afirma haber apoyado su creación y minimiza sus discrepancias con el nuevo ministro, Calvo-Sotelo deja ver que existieron roces entre ambos Departamentos (Oreja Aguirre, 2011, p. 197. Calvo-Sotelo, 1990, p. 145 y pp. 149-150). La correspondencia entre el Ministerio de Asuntos Exteriores y el de las Relaciones con las Comunidades, y diversos informes de Santa Cruz, demuestran cierta tensión, especialmente por el interés de Calvo-Sotelo de Ilevar en exclusiva la iniciativa política del proceso (Cfr. Nota informativa de la Dirección General de Relaciones Económicas Internacionales «Aspectos administrativos de las relaciones España-CEE». Madrid, 19 de julio de 1978. AMAE, caja 9, 14582). Otra, más importante, fue la salida de Oreja del Gobierno en septiembre de 1980. Algunos pensaron que había perdido la confianza del presidente por declararse en junio de ese año inequívocamente a favor de la entrada en la OTAN, pero la verdadera razón de su relevo por José Pedro Pérez-Llorca parece haber sido la presión que ejercieron en ese sentido Leopoldo Calvo-Sotelo y Francisco Fernández Ordóñez, hombres fuertes en esa remodelación ministerial (Fuentes, 2011, pp. 316-317 y p. 344; Martínez Sánchez, 2011, pp. 283-310).

La cuestión del ingreso en las Comunidades Europeas fue una de las vividas con mayor sintonía entre Adolfo Suárez y Leopoldo Calvo-Sotelo. La creación de un departamento para las Relaciones con las Comunidades Europeas en 1978 evidencia la prioridad que se dio al asunto. No obstante, el impulso para crear ese Ministerio vino del ámbito europeo: había sido lo habitual en los procesos de negociación de la incorporación de nuevos países. Todos los candidatos menos dos habían puesto en manos de un negociador con rango de ministro esa tarea (Calvo-Sotelo, 1990, p. 145). Parecía aconsejable hacer lo mismo, y se hizo. La comunicación entre el Ministerio para las Relaciones con las Comunidades Europeas y la Mon- 
cloa fue desde entonces fluida. Calvo-Sotelo hacía llegar a Adolfo Suárez informes periódicos sobre la evolución de las negociaciones, así como ideas para los discursos del presidente del Gobierno que tratasen aspectos de la cuestión europea. Se aprecia de manera singular en las notas que le envió de cara a la celebración del debate en el Congreso de los Diputados del 20 de mayo de 1980 (ALCS, Relaciones con la CEE, caja 100 , exp. 2).

Tomada la decisión, la elección de Leopoldo CalvoSotelo no fue inmediata. El de Ribadeo no había entrado en el Gobierno de 1977 porque, según le dijo Adolfo Suárez: «tengo que limpiar tu imagen». El presidente no especificó las razones de esa necesidad. Calvo-Sotelo supone que eran las tareas que había debido realizar para aunar los distintos grupos en UCD, y también «un reproche al gerente de la campaña por no haber obtenido mayoría absoluta» (Calvo-Sotelo, 1990, p. 58). Suárez valoró para el nuevo Ministerio los nombres de Federico Mayor Zaragoza, Rafael Calvo Ortega, Jaime Lamo de Espinosa o José Lladó (Bassols, 1995, p. 204). En febrero de 1978, no obstante, Suárez decidió que el hombre indicado para la tarea de dirigir esa negociación era Leopoldo Calvo-Sotelo. Según algunos testigos tuvo pocas o ninguna duda al respecto (Entrevista con Raimundo Bassols, 14/12/2011). Pero que el presidente confiara tanto en él no significa que no hubiera diferencias entre los dos centristas.

Una primera se refiere al lugar que las relaciones exteriores ocupaban en la agenda de uno y otro. La cuestión del ingreso en las Comunidades no parecía contarse entre los temas de atención preferente para Adolfo Suárez, más centrado en asuntos de política doméstica. Leopoldo Calvo-Sotelo recuerda que era frecuente que el presidente se ausentara del Consejo de Ministros cuando él informaba de la marcha de las negociaciones, y subraya que solo en una ocasión consiguió llevarlo a Bruselas, invitado por el presidente de la Comisión, Jenkins. Desgraciadamente el líder centrista se sintió allí "fuera de lugar, aislado» (Calvo-Sotelo, 1990, p. 126).

Esta diferencia influía en otro punto interesante, una cuestión de perspectiva que Calvo-Sotelo resume así:

«La pertenencia a la Comunidad Europea era más sustantiva, pero el ingreso en la Alianza Atlántica fue más definitorio de nuestro posicionamiento exterior» (Calvo-Sotelo, 1999, p. 140).

La OTAN, en efecto, fue uno de los dos puntos clave para de la Transición política exterior. Pero a diferencia de la cuestión de las Comunidades, la Alian- za era mucho más polémica: no existía ni entre los grupos políticos ni en la opinión pública la práctica unanimidad con que contaba la entrada en el Mercado Común, sino que una parte de la clase política, casi toda la izquierda, se oponía al ingreso de España en la Organización Atlántica. Esto planteaba una cuestión de oportunidad que precisaba decisiones y se dilató con indecisiones. En esto Suárez y CalvoSotelo no coincidían.

¿Por qué se tardó en proponer la entrada de España en la OTAN y no se hizo a la par de la solicitud en la Comunidad? Para responder a esa pregunta CalvoSotelo recordaba cómo la posible solicitud de entrada en la Organización Atlántica había aparecido en repetidas ocasiones en los programas electorales de UCD, quedándose en promesa incumplida (Conferencia «La Transición política exterior», Barcelona, 18 de diciembre de 1985, ALCS, caja 35, exp. 3). Una de las razones que lo explican, a su juicio, fue el escaso afecto mostrado hacia España por quienes, en teoría, deseaban una rápida democratización del país que permitiese incluirlo en el seno de las organizaciones internacionales. Esa actitud, que no se podría llamar hostilidad, acentuó sin embargo el predominio de la política interior en las preocupaciones y sentimientos de la clase política y, de manera evidente, en Adolfo Suárez, un presidente que nunca se encontró a sus anchas fuera de España o, por ser más precisos, del ámbito hispano. De ahí su predilección por los viajes al continente iberoamericano en detrimento de otros países que integraban las Comunidad; de ahí su participación como país observador en la Cumbre de los No Alineados, celebrada en La Habana en 1978. No había en esto, sin embargo, un ánimo revolucionario o esencialmente antiamericano, más bien era otra cosa:

«el Suárez que enviaba una delegación de alto nivel a la Conferencia de los No Alineados y se mostraba ambiguo sobre el tema de la OTAN, no quería transmitir ningún mensaje especial más allá del muy español y castizo hacer-lo-que-dicta-la-real-gana» (Calvo-Sotelo, 2005, p. 67).

Pero, como veremos, había más motivos en los que, probablemente, Calvo-Sotelo no consideró oportuno insistir en sus memorias.

La ambigüedad en la política atlantista de UCD se puede enmarcar dentro de la ambigüedad general que caracterizó a la formación política, fuera de dos ideas clave: la de llevar a España hacia la democracia mediante una reforma pactada de la Ley a la Ley y la de la entrada de nuestro país en las Comunidades 
Europeas. Y en esto sí desempeñó un papel importante el criterio de Adolfo Suárez, especialmente a partir de 1979, cuando la complicada escena internacional y las persistentes dificultades internas llevaron al presidente a interesarse cada vez más por los asuntos internacionales. Juan Francisco Fuentes, en su excelente biografía del líder centrista ofrece una detallada visión del pensamiento en política exterior de Adolfo Suárez que coloca bajo el epígrafe «Entre Washington y Ormuz». El título se refiere al Ilamado Síndrome del estrecho Ormuz, como sus íntimos se referían a una de sus ideas más originales, elaborada por aquellas fechas mientras contemplaba un globo terrestre en su despacho. La idea consistía en prestar una nueva atención, prioritaria, a Oriente Medio en un momento de intenso cambio -invasión soviética de Afganistán, revolución islámica iraní, guerra IránIrak - que reclamaba una acción original y decidida en la zona. El primer paso, según Suárez, era conceder protagonismo a Yasser Arafat, como él mismo había hecho al recibirlo, para encontrar solución al problema palestino y caminar decididamente hacia la pacificación de esa tierra.

En su viaje a Estados Unidos en enero de 1980 Suárez expuso al presidente norteamericano Jimmy Carter sus ideas al respecto, y también las que tenía sobre Centroamérica, concretamente sobre Nicaragua, y el potencial papel mediador de España (Fuentes, 2011, pp. 311-312). Calvo-Sotelo sostiene en sus memorias que la hostilidad hacia Occidente de Suárez se acentuó cuando Carter no hizo caso de su parecer (Calvo-Sotelo, 1990, p. 117).

En opinión de Fuentes tanto o más fundamental que lo anterior en la postura de Suárez en 1979-1980 era su creciente prevención ante la Unión Soviética. Pensaba que estaba detrás de ETA y temía que la aprobación de la entrada en la OTAN generara una oleada de atentados (Fuentes, 2011, pp. 313-314). Así

«El antiamericanismo, por un lado, y el anticomunismo, por otro, abocaban a una política exterior neutralista o tercermundista, como se dijo entonces sin intención peyorativa» (Fuentes, 2011, p. 315).

Un planteamiento bien distinto, pues, del que se derivaba de las ideas y los planes de Leopoldo Calvo-Sotelo.

Ya hemos dicho que Calvo-Sotelo impulsó la llegada de Pérez-Llorca al frente de Exteriores. Pues bien, al poco tiempo de ocupar el cargo, en octubre de 1980, el nuevo ministro convenció a Suárez de que convenía no prolongar más la decisión sobre la en- trada en la OTAN. Suárez refrendó su acuerdo en una carta: "Adelante, prepáralo todo en secreto» (Fuentes, 2011, p. 317. Cita de Alonso-Castrillo, 1996, pp. 462-463 у p. 471). Hubo decisión, pues, pero sin abandonar la ambigüedad pública.

Pocos meses más tarde, cuando Leopoldo CalvoSotelo se hizo cargo de la Presidencia del Gobierno la actitud ante estas cuestiones cambió sensiblemente. Era lógico en un Gobierno que tuvo por cabeza alguien que prestaba una atención especial y directa a estos asuntos, invirtiendo en buena medida el razonamiento de Suárez: Leopoldo Calvo-Sotelo miraba la política exterior como manifestación del proyecto interior, no en cuanto ayuda u obstáculo para la política doméstica.

\section{EL GOBIERNO CALVO-SOTELO. LOGROS Y PROYECTOS INCONCLUSOS}

«(...) Yo tenía mis ilusiones (...). Una de ellas era el cambio económico (...). Otra definir la política exterior, porque yo, como ministro, he tenido la vivencia inmediata de que España era un país que no tenía una línea de política exterior, porque no la ha tenido nunca desde Carlos III. Pero nadie se preocupa de eso. Se preocupaban de otras cosas. Los españoles estábamos en una neutralidad forzosa» (Prego, 2000, pp. 124-125).

Leopoldo Calvo-Sotelo llegó a la Presidencia del Gobierno con una fecha subrayada en su calendario: marzo de 1983, límite inaplazable de la I legislatura. Tenía pues, antes del veredicto de las urnas, a lo sumo dos años de mandato. Era evidente, más aún teniendo en cuenta los cada vez más preocupantes síntomas de crisis interna de la UCD, la necesidad de jerarquizar las medidas. Si bien es cierto que el intento de golpe de Estado - en pleno proceso de investidura - tuvo que modificar el orden de prioridades, el proyecto exterior del Gobierno Calvo-Sotelo se mantuvo en buena medida idéntico al anunciado en su discurso ante el Parlamento el 19 de febrero de 1981. Dos eran las medidas claves en este apartado: el ingreso en la OTAN y en la CEE, organizaciones que conformaban en la mente de Calvo-Sotelo una unidad a la que España debía adherirse, sin más dilaciones ni dudas, para reintegrarse en el mundo occidental. En primer lugar porque era al que por historia y cultura debía pertenecer y, en segundo, para dar un salto de calidad en la economía, tan vinculada ya, de hecho, con las de los países del Mercado Común. Las Comunidades Europeas podían significar el factor clave en la modernización española, especialmente en la difí- 
cil etapa de crisis económica que sufría el país desde finales de la década de los setenta.

Durante sus años en la Moncloa, la negociación con el Mercado Común no se desarrolló al ritmo que quería España, mucho más rápido que el que se vivió, sino al que permitieron los problemas internos comunitarios. Un rápido repaso por la sucesiva lista de sesiones negociadoras da cuenta de su cantidad y de su denso contenido (Bassols, 1995, pp. 341-342). En año y medio, entre marzo de 1981 y octubre de 1982, se desarrollaron 6 sesiones a nivel de ministros, con presencia del titular de Exteriores, PérezLlorca: de la del 17 de marzo de 1981, en la que se trataron, entre otros, asuntos relacionados con el derecho de establecimiento, la unión aduanera, las relaciones exteriores... a la del 26 de octubre de 1982, que versó sobre la unión aduanera y la CECA. Hubo, además, 11 sesiones a nivel de suplentes, encabezadas por parte española por el secretario de Estado, Raimundo Bassols, que inició en septiembre una gira por las capitales europeas. Es decir, en conjunto una media de una reunión mensual.

El presidente Calvo-Sotelo intervino también, y de manera directa. Recibió al cuerpo diplomático de los países comunitarios en marzo de 1981, presidió la Comisión Delegada de Asuntos Exteriores el 26 y el 30 de junio de ese mismo año, y realizó una serie de visitas oficiales a los países europeos en los que la consecución de la adhesión a la Comunidad y el futuro de Europa, eran tema imprescindible: en abril del 1981 visitó Bonn; en julio, París; en enero de 1982, Bruselas y Londres; en marzo, Roma; en abril, Atenas; en noviembre, Copenhague. A la vez, recibió en España las visitas del presidente francés François Miterrand o el vicecanciller germano Hans-Dietrich Genscher.

El ritmo de la negociación y la esperanza de acelerar su final, permitía a los negociadores españoles confiar en la fecha del 1 de enero de 1984 como la de la entrada de España. Así lo afirmaba Bassols en declaraciones El Correo Catalán en abril de 1981. (Ministerio de Asuntos Exteriores Actividades, Textos, Documentos, editado por la Oficina de Información Diplomática, Madrid, 1982). Dos meses más tarde, el 29 de junio, Bassols escribía a Matías Rodríguez Inciarte, Secretario de Estado Adjunto al presidente Calvo-Sotelo: «La negociación está razonablemente avanzada, salvo en lo que respecta al capítulo de pesca y al capítulo agrícola (...). Técnicamente resultaría posible que si los dos capítulos bloqueados empezasen a ser tratados libremente y en profundidad a partir del mes de Septiembre la negociación terminase en Julio del 82 o máximo en otoño del 82, lo que permitiría mantener en pie el programa de entrada en vigor del Acuerdo el 1 de Enero de 1984». (ALCS, caja 100, exp. 11).

La opinión en Europa, en cambio, no era uniforme. Mientras en el mismo mes de abril del 81 Jacques Chirac alertaba en El País de que la entrada de España podía paralizar la CEE, el vicecanciller alemán Genscher afirmaba que la incorporación de España a la CEE era irreversible. A pesar de que el comisario Lorenzo Natali urgía, en noviembre del mismo año a la comisión de agricultura del Parlamento Europeo a que se acelerasen las negociaciones agrícolas con España, de modo que la adhesión pudiera realizarse el 1 de enero de 1984, la propia Comisión - por petición francesa- encargó un nuevo informe, otro más, sobre los problemas que podría plantear el ingreso de España y Portugal en el Mercado Común CEE.

A pesar del empeño del equipo negociador, la adhesión al Mercado Común fue un proyecto inconcluso. La oposición de Francia, país determinante entonces en la CEE (Calvo-Sotelo, 2005, p. 57), pesó más que la retórica europeísta que tantas oportunidades había prometido a España cuando fuese una democracia.

La otra cara de la moneda, la incorporación a la OTAN, pudo culminarse, en cambio, con éxito, a pesar de la fuerte resistencia de la oposición que veía la medida como una ruptura del statu quo y de la tradicional política neutralista de España. Esa era, justamente, una visión política que Calvo-Sotelo, quería remover: España debía dejar claro su anclaje europeo y occidental. Por ello en el discurso de investidura había señalado que

«el Gobierno que aspiro a presidir reafirma su vocación atlántica, expresamente manifestada por la Unión de Centro Democrático y se propone iniciar las consultas con los Grupos Parlamentarios a fin de articular una mayoría, escoger el momento y definir las condiciones y modalidades en que España estará dispuesta a participar en la Alianza»

(Calvo-Sotelo, 1982, p. 22).

En este caso lo más laborioso fue el camino en las Cortes españolas. Comenzó un largo recorrido parlamentario, en el que el Gobierno puso de manifiesto su empeño de diálogo y resolución, recorrido que concluyó, efectivamente, con el ingreso de España en la OTAN. Los hitos más importantes del proceso fueron los siguientes. El Consejo de Ministros acordó, el 20 de agosto de 1981, solicitar del Consejo de Estado dictamen sobre la adhesión de España a la OTAN, a efectos de su ulterior tramitación parlamentaria. Al 
día siguiente el presidente del Gobierno, acompañado de los ministros de Asuntos Exteriores y Defensa, se reunió con la Junta de Jefes de Estado Mayor a quienes explicó las razones que motivaban la decisión de solicitar el dictamen. Ya en sede parlamentaria, el 15 de septiembre, el pleno del Congreso decidió que la Comisión de Asuntos Exteriores era la competente para tramitar el proyecto de adhesión de España a la OTAN²; entre el 6 y el 8 de octubre la Comisión de Asuntos Exteriores del Congreso aprobó el proyecto de resolución sobre la adhesión a la OTAN presentado conjuntamente por UCD y Coalición Democrática. EI 29 de octubre el pleno del Congreso de los Diputados aprobó la petición presentada por el Gobierno para negociar la integración de España en la $\operatorname{OTAN}^{3} \mathrm{y}$, por fin, el 26 de noviembre el pleno del Senado ratificó la decisión del Congreso de autorizar al Gobierno para que negociara la adhesión de España a la OTAN ${ }^{4}$.

Cumplidos los trámites necesarios, el 2 de diciembre el embajador español en Bruselas, Nuño Aguirre de Cárcer, entregó al secretario general de la OTAN la carta por la que el Gobierno se declaraba dispuesto a recibir la invitación de ingresar en a OTAN, que fue contestada con un comunicado en el que la Alianza recibía oficialmente y acogía favorablemente la disposición de España, y anunciaba la puesta en marcha del proceso de adhesión. A partir de entonces, en todos los Parlamentos de los países miembros se deliberó sobre el necesario respaldo a la adhesión, que se inició el 3 diciembre con votación en el Parlamento holandés y concluyó el 24 de mayo de 1982 con la ratificación del griego.

El 30 de mayo España pudo depositar en Washington el instrumento de adhesión a la OTAN, convirtiéndose en su miembro número 16 . El 10 de junio de 1982 Leopoldo Calvo-Sotelo asistió como presidente del Gobierno de España, por vez primera, a la cumbre de Jefes Estado y de Gobierno de la Alianza Atlántica. Le había costado 15 meses a la cabeza del Ejecutivo hacer cumplir su compromiso. Pero cuando se conoce su pensamiento sobre la política exterior se entiende cómo a la satisfacción por este logro debía ir unida la frustración por la marcha de las negociaciones con la CEE. Lo que él llamaría transición exterior se estaba demostrando más difícil de lo esperado.

\section{LA OTAN Y «LA CONTUMACIA DEL PSOE»}

«Quienes en 1976, en torno a Adolfo Suárez, aceptamos sobre nuestros hombros el primer Gobierno de la Transición, estábamos convencidos de que la que ahora llamo Transición Interior iba a ser más ardua y lenta que la Transición Exterior. Nos equivocamos. (...) ¿Por qué este retraso de la Transición Exterior respeto de la Interior? En buena parte por la contumacia del PSOE» (Conferencia «La Unión Europea se amplía sin precisar su naturaleza», Oviedo, 15 de marzo de 1994. ALCS).

La OTAN fue un punto clave para la transición política exterior. Y, a diferencia de la adhesión en la CEE, mucho más polémico dentro de España: no existía la unanimidad con que contaba el ingreso en el Mercado Común, sino que una parte del arco parlamentario, toda la izquierda, se oponía al ingreso de España en la Organización Atlántica.

Fue Calvo-Sotelo, desde la Presidencia del Gobierno, el que terminó con la indeterminación en favor de un claro alineamiento con el bloque occidental y democrático. Fue una restitución histórica que podía haber concluido en 1982, con la ratificación en las Cortes del ingreso en la OTAN, pero fue retrasado por la decisión del nuevo ejecutivo socialista.

Probablemente por ello, Calvo-Sotelo se mostró especialmente crítico con la actitud de los socialistas que, a su juicio, bloquearon innecesariamente el anclaje occidental de España. Una crítica, además, que se entiende mejor sumándola a la acusación de reescritura de la Historia que nuestro protagonista endosaba al Gobierno socialista. Así aparecía a sus ojos el afán del Gobierno González de apuntarse prácticamente en exclusiva la adhesión de España al Mercado Común y el fin de la polémica sobre la Organización Atlántica. Es un hecho que el PSOE concluyó las negociaciones en 1985 y firmó la adhesión de España a las Comunidades en 1986, pero también lo son las largas horas de negociación y trabajo de los equipos negociadores de la UCD. No obstante, la segunda parte de la afirmación, el fin de la polémica en torno a la OTAN, era la más discutible. El ex presidente lo sabía y, por ello, formuló la siguiente tesis: el Partido Socialista fue, básicamente, partidario de la neutralidad española en la cuestión OTAN y, por extensión, en la cuestión de los bloques. Por ello manifestó su oposición a la entrada de España, prometiendo un referéndum si llegaba al poder, en el que se entendía que votarían no. No sería sino hasta su entrada en el Gobierno cuando su posición cambiaría (Conferencia "La Transición política exterior», Barcelona, 18 de diciembre de 1985, ALCS, caja 35, exp. 3).

Pero fue un cambio precedido de otro: cuando se creó la Alianza Atlántica en 1949, impulsada por la pugna aliado-soviética en torno al futuro de la Alemania dividida y el bloqueo de Berlín, el Partido Socialista en el exilio (Indalecio Prieto, Rodolfo Llopis, Luis Araquistáin...) se había manifestado a favor del ingre- 
so de España en la organización, una vez derrocado el franquismo (Calvo-Sotelo, 2005, p. 63; Gillespie, 1991, p. 129; Míguez, 1989, pp. 157-162). A partir del congreso de Suresnes, la posición varió, y los congresos socialistas de los años 1974, 1976, 1979 y 1981 definieron su oposición a cualquier organización basada en la hegemonía y que pusiera fin a la neutralidad española. En el congreso de 1984 - con los socialistas ya en el poder - se observó el inicio de un nuevo cambio: ya no se ponía en tela de juicio la organización en sí, sino la forma en que UCD había decidido la entrada de España, a juicio de los socialistas, «irreflexiva, precipitada y gratuita, rompiendo el consenso» (Conferencia «La Transición política exterior», Barcelona, 18 de diciembre de 1985, ALCS, caja 35, exp. 3; Andrade Blanco, 2007, pp. 97-106). Era esta una acusación que -dándose por aludido-Calvo-Sotelo quiso rebatir:

«¿̇Irreflexiva? Según el propio Presidente del Gobierno ha dicho en unas declaraciones al diario el País hace unas semanas, su reflexión durante esos dos o tres años le ha llevado a creer que es buena la presencia de España en la Alianza Atlántica; es decir, su reflexión le ha llevado a la misma posición que a mí me llevó la mía; es decir, la que no fue reflexiva fue la actitud del PSOE en 1981.

„¿Precipitada? Una decisión que está en documentos del Partido desde 1977; que se anuncia formalmente por mí en febrero del 81 (anoto como nota al pie de página que se anuncia el 18 de febrero, antes del 23, y digo esto porque también se ha afirmado que entramos en a alianza como consecuencia del 23 de febrero), que el Gobierno decide presentar al Congreso el 20 de agosto; que se debate en seis larguísimas sesiones, en comisiones y en pleno, en el Congreso y en el Senado en octubre y noviembre del 81; y que se perfecciona en mayo del 82, ¿puede llamarse una decisión precipitada? Claro que luego la cuestión se ha venido arrastrando durante tres años en una penosa marcha hacia la ética de la responsabilidad, desde la ética de la convicción (como en aquellas declaraciones a El País dijo el Presidente del Gobierno, leyendo muy deprisa a Max Weber) (...).

»Es la actitud del PSOE en el 81 la que ha resultado irreflexiva, precipitada y gratuita» (Conferencia "La Transición política exterior», Barcelona, 18 de diciembre de 1985, ALCS, caja 35, exp. 3.).

A su juicio, la clave de la postura neutralista del PSOE en esta cuestión eran los apoyos internacionales con los que contaba el partido, la opinión sobre la OTAN de las figuras clave que habían ayudado a los socialistas desde el inicio de la Transición y, concretamente, Willy Brandt, Bruno Kreisky y Olof Palme. Todos estos políticos habían vivido en unas condiciones muy distintas a las españolas. Según Calvo-Sotelo no era comparable la oportunidad histórica que tenía España con el nacimiento del régimen democrático con la posición del canciller de la Alemania Occidental en plena Ostopolitik. Ni tampoco con la de los políticos austriaco y sueco que vivían en dos naciones ancladas en un neutralismo que no deseaba asociarse a ninguna institución internacional. Y fue esa influencia la que conformó la postura socialista, una postura que, con el paso del tiempo, tuvo que variar. Y así Felipe González, desde el poder, convocó el prometido referéndum... pero para votar que sí. Esa fue la razón que le permitió jactarse de haber convencido a una población no partidaria de la OTAN... precisamente porque el PSOE se pasó años fomentando esa postura (Martínez Sánchez, 2011, pp. 283-310). Sea como fuere, finalmente, venció el sí. Con ello, y con la entrada oficial de España en la CEE el 1 de enero de 1986, la Transición política exterior quedó, por fin, sellada.

"La contumacia» del PSOE» en la cuestión de la OTAN tiene, pues dos vertientes, en el pensamiento de Calvo-Sotelo: por un lado, la oposición efectiva a su Gobierno que en torno a esta cuestión realizaron los socialistas en 1981-1982 y, por otro, el análisis a posteriori de su política en torno a este asunto. Atribuyendo a UCD y al Gobierno Calvo-Sotelo una irresponsabilidad en la que no habían incurrido, los socialistas venían a negar su propia responsabilidad en torno a un asunto de evidente interés de Estado que parecía más bien tratado en clave ideológica o de partido. Al fin, el repaso de los acontecimientos y los datos de toda la polémica atlántica, cerrada finalmente en 1986, parecen dar la razón al Gobierno Calvo-Sotelo en torno a la oportunidad del ingreso de España en la organización y el fin práctico de la política neutralista, pues fue esa finalmente la postura que, pragmáticamente, aceptó tiempo después el Gobierno González.

\section{VALORACIONES EN PERSPECTIVA}

Tras haber participado activa y destacadamente en la vuelta de España a los foros internacionales que le correspondían, Leopoldo Calvo-Sotelo se convirtió en un analista político, interesado en las cuestiones exteriores y, de forma específica, en Europa y el Mercado Común. El paso del tiempo y el fin de sus responsabilidades públicas, le permitió la libertad y perspectiva necesarias para ofrecer una valoración de lo que supuso y ha supuesto la política exterior durante la Transición, en la que tuvo un papel protagonista. 
Una de las principales ideas que destacan en sus reflexiones fue el acierto que supuso el acercamiento a Europa. España necesitaba a Europa, y Europa no estaba completa sin España. En este sentido pudo afirmar que el proceso exterior de la Transición tuvo el mismo alcance y trascendencia que el interior, aunque este haya tenido más reconocimiento con el paso del tiempo y haya sido la mayor preocupación de los sucesivos Gabinetes.

«En el umbral de la Transición - dejó escrito- aparecen juntas, con el mismo rango, la reconciliación, la democracia y la nueva política exterior. Reconciliación y democracia llegarían de la mano de Adolfo Suárez, en un plazo increíblemente corto, entre 1977 y 1978. La política exterior se haría esperar bastante más, hasta 1981» (Calvo-Sotelo, 1999, p. 138).

A su juicio, el balance de España como miembro comunitario no podía valorarse sino positivamente, por dos razones. La primera, política: tras el ostracismo internacional vivido durante siglos, España pudo por fin situarse como uno más en Europa, considerándose de nuevo lo que geográfica y culturalmente nunca había dejado de ser: un país plenamente europeo. La segunda, económica: la adhesión de España a la CEE, aunque generara algún inconveniente, fue claramente beneficiosa (Calvo-Sotelo, 1999, p. 35).

Sin embargo, la larga espera para entrar en las Comunidades Europeas, de las que se esperaba una rápida modernización y homologación con el resto de países miembros, junto con la esperanza de un rápido crecimiento económico, produjo entre los españoles una creencia europeísta hasta cierto punto exclusivamente sentimental, que con el paso del tiempo dio lugar a un cierto grado de frustración y escepticismo en torno a la construcción europea. Calvo-Sotelo achacaba este desencanto a las inmensas expectativas que se habían generado, así como a cierto desánimo producido por la larga negociación de adhesión. Sus reflexiones en este punto se teñían de una matizada nostalgia. En esa larga espera los españoles y su Gobierno habían podido comprobar cómo a la lógica política y al deseo de que España se reintegrase en el mundo europeo, los países comunitarios antepusieron sus intereses particulares, de carácter principalmente económico: con la entrada de un nuevo país, de tamaño considerable, había que repartir de nuevo costes y beneficios:

«Entramos los españoles en la ardua negociación del siglo XX con la esperanza ingenua de que en esos foros nos recibirían con los brazos abiertos porque llegábamos desde el frío de un aislamiento forzoso y muy largo; que iban a degollar el ternero cebado, como en la parábola del hijo pródigo para la gran fiesta de nuestro recibimiento. Pero muy pronto hubimos de comprobar, por nuestra cuenta y a nuestras costas, lo que otros sabían ya: que la comunidad internacional es todo menos evangélica, que tanto en la Unión Europea in fieri como en la Alianza Atlántica ya madura, y muy especialmente en la Unión, los juegos ya estaban hechos, las ventajas repartidas entre los socios antiguos y que nadie estaba dispuesto de buen grado a mover su silla en la mesa redonda común para hacer un sitio en ella al recién llegado» (Calvo-Sotelo, 2005, pp. 53-54).

Es interesante la mención de la OTAN pues, habitualmente, se ha considerado que sus miembros y, de forma destacada, Estados Unidos deseaban con cierto grado de impaciencia y entusiasmo la entrada de España, pero en realidad era nuestro país el primer interesado, pues a los americanos lo que más les preocupaba era la renovación de los acuerdos bilaterales (Calvo-Sotelo, 1999, p. 93). (Cfr. Powell, 2011, p. 556, p. 570 y p. 593, donde el ministro socialista de Asuntos Exteriores, Fernando Morán, expresa la misma opinión).

En este sentido merecen destacarse otras dos ideas que el ex presidente no pudo manifestar, al menos con total sinceridad, mientras ejerció funciones públicas: la primera, la supuesta relación entre la entrada en la CEE y la OTAN; la segunda, sobre el papel de Francia en nuestra transición exterior.

Una de las acusaciones más repetidas contra el Gobierno de UCD por parte de los opositores a la OTAN era la relación inseparable entre el Mercado Común y la Alianza: aunque a nuestro país solo le convenía y necesitaba la entrada en la CEE, se nos obligaba también a pedir la adhesión a la Alianza. Ciertamente se podía establecer una relación entre ambas organizaciones, al fin y al cabo propias de los países occidentales y democráticos, aunque los Gobiernos de UCD fueron especialmente cautos en distinguir y separar ambos procesos. Es obvio, por otro lado, que la afirmación de que solo se buscaba la entrada en la OTAN para favorecer el ingreso en la CEE además de inexacta podía ser claramente perjudicial para los intereses de España; pero no es posible obviar que la entrada en la Alianza Atlántica favoreció más que perjudicó la negociación a la CEE y pudo ser usada como una muestra de la seriedad y responsabilidad internacional de la nueva España democrática. 
La percepción desde el extranjero de cómo se gestionaba esta cuestión coincide a grandes rasgos con esa versión:

"Spain's entry into NATO was motivated primarily by political factors rather than military or security concerns, Prime Minister Leopoldo Calvo-Sotelo sought to achieve a number of objectives by bringing his country into the alliance. First, Spain would finaIly have a voice of its own in European affairs, moving away from its period of exile that resulted from the years of Francisco Franco. Second, entry into NATO would support Spain's application into the European Economic Community (EEC). Spain had first applied for membership in 1977, and it appeared that the EEC was dragging its feet on the request. Third, the government believed that Spanish entry would speed up the negotiating process with Great Britain on the future status of Gibraltar» (Allin, 1985, p. 62).

En la serie de entrevistas que la periodista Victoria Prego realizó en 2000 a los cuatro presidente de la democracia se planteó esta cuestión: Calvo-Sotelo confirmó entonces esa idea: la Alianza era necesaria en sí misma, pues suponía un punto y aparte en la trayectoria política exterior de España aunque, además, pudiera contribuir a acortar el largo camino de negociación que la Comunidad estaba imponiendo a España (Prego, 2000, p. 158).

La segunda reflexión incide en que si hubo un país que contribuyó de manera destacada a ralentizar el ritmo de la adhesión española, ese fue Francia, que temía la competencia agrícola con España. Esta idea estaba presente desde el mismo comienzo de su etapa como ministro para las Relaciones con las Comunidades Europeas. Un informe, elaborado tras el primer viaje de Calvo-Sotelo a París, afirmaba:

«Francia no ha deducido todavía las consecuencias naturales de la existencia al otro lado de los Pirineos de una país democrático y desarrollado. España sigue siendo piedra de toque interior francesa, y divide tanto a la mayoría como a la izquierda: los adversarios de la adhesión de España están radicalizados por la crisis económica y por la propaganda electoral: los amigos no se han despojado aun de una cierta propensión a la tutela. Se echa en falta un planteamiento nuevo por parte española de las relaciones con Francia, como corresponde a la nueva situación española». (Nota para el señor Presidente del Gobierno sobre viaje a Francia. Madrid, 9 de junio de 1978. ALCS, Relaciones con la CEE, caja 69, exp. 3).
Especialmente delicada fue la postura de Giscard d'Estaing que, tras ejercer como patrocinador de la nueva España en la coronación del rey Juan Carlos I, ralentizó más adelante, deliberadamente, el ritmo de la negociación española. "La realidad era bien distinta en aquellos años: la realidad era que Francia no quería la entrada de España a ningún precio» (CalvoSotelo, 1990, p. 166).

«[Mi experiencia] de negociador durante tres años con la Comunidad me dejó una imagen de Francia —de la Francia de V[aléry] G[iscard] [d']E[staing]como obstáculo» (Calvo-Sotelo, 1999, pp. 178-179).

El análisis objetivo que solo el tiempo otorga permite, en ocasiones, matizar o cambiar las ideas que se han manifestado sobre un suceso. En el caso del ex presidente esta distancia le sirvió por un lado, para confirmar la necesidad y el acierto de acercarse a Europa, recién recuperada la democracia en nuestro país; y por otro, para hacer balance y expresar con la libertad que solo tiene el «cesante», juicios sobre actitudes o personas; juicios que si en otro momento podían perjudicar a España, ahora servían en su opinión para poner orden en los hechos históricos y valorar con más precisión nuestra historia reciente.

\section{CONCLUSIONES}

Merece la pena subrayar, en primer término, la continuidad de las convicciones europeístas de Leopoldo Calvo-Sotelo: fue una referencia orientadora desde su juventud, desde sus balbuceos políticos, hasta el final de su actividad pública. El enraizamiento orteguiano de sus ideas en esta materia y su interés por conocer directamente qué significaba la construcción europea vinculada a la idea comunitaria terminaron por fortalecer esa inicial convicción suya, hasta convertirla en elemento central de su actividad política y parte esencial de su programa como presidente de Gobierno.

Además, la idea que Leopoldo Calvo-Sotelo se hizo de la historia de España fue determinante en su modo de entender cuáles debían ser las metas exteriores de la Transición. Quizá lo más importante de su visión fuera que el cambio en política exterior era tan importante como en la interior; no era menos necesario ni un adorno, sino esencial, en el sentido de que debía superarse una época de nuestra historia en la que España había vivido desencajada de su contexto, como ausente de Europa y, por eso, del mundo. Esa época no era solamente el régimen de Franco, tenía según él una antigüedad de dos siglos, tanta como el tiempo que hacía que España carecía de voz autorizada en 
el ámbito internacional, voz que le correspondía por lo que históricamente había sido y debía volver a ser como elemento integrante de Europa y Occidente.

Su proyecto de devolución de España a su lugar en el concierto internacional se concretó en superar dos disfunciones que consideraba graves: el neutralismo y la ausencia del proyecto de construcción europea.

El programa para conseguir esa meta miraba lo económico como una dimensión más, ciertamente de entidad, pero nunca la única ni la más importante del problema. Por eso un acuerdo económico preferencial con la Comunidad Económica Europea era algo claramente insuficiente, y la incorporación a las Comunidades Europeas era crucial, pero no lo era todo. Ese paso, que pretendió adelantar todo lo que pudo, debía ir acompañado de la entrada en la Alianza Atlántica y de algunas cosas más. Porque, con ser importantes esos objetivos, no dejaban de ser el medio para empezar a hacer una nueva política exterior, que llevara a España a intervenir en los asuntos internacionales como lo hacían sus pares, los demás países europeos y occidentales, en los foros institucionales creados para ejercerla.

La comparación con la actitud de Adolfo Suárez ante estos asuntos pone de relieve cómo los líderes de la UCD tuvieron prioridades diferentes en este asunto. Para Adolfo Suárez la normalización exterior era un corolario de la interior, importante, pero no esencial. Los dos presidentes centristas de la naciente democracia coincidieron en los fines principales de la política exterior, pero por diferentes motivos. En Suárez prevalecieron las consideraciones de política interior y condicionaron su administración del tiempo en las decisiones de política exterior. En el político abulense encontramos, además, rasgos de intuición visionaria, atentos a las oportunidades de cambio en el presente y futuro próximos que no se dan en Calvo-Sotelo, modelado por su reflexión acerca de los hechos históricos, menos dado a imaginar escenarios inéditos y convencido de la importancia de las decisiones de política exterior en sí mismas. Es más, en él prevalecía la ponderación de las consecuencias interiores que tenía el actuar o abstenerse en las relaciones exteriores. La consecuencia más relevante de esas diferencias fue el retraso del proceso de incorporación a la OTAN, que se agilizó cuando Leopoldo Calvo-Sotelo asumió la presidencia del Gobierno.

Algo parecido se observa al comparar la actitud del Partido Socialista Obrero Español y de Felipe González en esta materia. Asumida la conveniencia de incorporarse a las Comunidades Europeas como un lugar común, la oposición socialista encontró en el neutralismo un argumento de disensión que parecía más basado en condicionamientos de táctica electoral que de sentido de Estado. Era en buena medida lógico: no tenían experiencia de Gobierno, y buscaban el camino para acceder a él. No se aprecia en ellos interés por la historia de las decisiones al respecto ni en el propio partido ni en el pasado español en general. Casi toda su argumentación parece centrada en olvidarlo para crear algo diferente. Puede que para algunos de ellos este fuera uno de los elementos centrales de la "ruptura» a que aspiraban.

La historia de la política exterior en la Transición pone, en definitiva, ante un interesante panorama de cómo se entrecruzan referencias históricas y políticas. Leopoldo Calvo-Sotelo, uno de los hombres que más energías le dedicaron, es un ejemplo de hasta qué punto la Transición fue una empresa que aunó continuidad y cambio, y no solo en términos de siglo XX, o de dictadura y democracia, sino en términos más amplios, de posición estratégica de nuestro país en el tablero internacional. El calado intelectual del planteamiento político de Leopoldo Calvo-Sotelo, su conocimiento directo de la realidad europea, su familiaridad con nuestra historia, fueron probablemente la causa de que su mirada abarcara un arco más amplio y se moviera en un plano más elevado que la táctica política preocupada de la toma o conservación del poder a corto plazo. Quizá por eso él sea de los pocos políticos que mencionan como precedentes importantes cambios producidos durante el régimen de Franco en esta materia, elemento que subraya lo que la Transición tuvo de continuidad además de ruptura. Es una importante realidad sin la que es muy difícil comprender la Transición, querida y dirigida por españoles educados durante el franquismo que habían puesto en el horizonte europeo su mirada, a veces desde su juventud.

Esa amplitud de visión característica de Leopoldo Calvo-Sotelo, producto de su cultura y de su experiencia profesional y política, hizo de la suya una de las miradas más europeas sobre los asuntos españoles, en contraste con la fuerte tendencia hispanocéntrica de la mayor parte de nuestros políticos de entonces.

\section{AGRADECIMIENTOS}

El Archivo de Leopoldo Calvo-Sotelo Bustelo se cita en el texto como ALCS. Se ha tenido acceso a ese archivo personal con la expresa autorización de su familia, a la que agradecemos su disponibilidad y confianza. El Archivo del Ministerio de Asuntos Exteriores como AMAE y el Archivo General de la Administración como AGA. 
1 «La actual estructura del Departamento es la de un equipo reducido, altamente especializado y, como ha dicho Leopoldo Calvo-Sotelo, sin ser una "verdadera estructura ministerial ni sustituir a los diversos Departamentos de la Administración". Se trata de un pequeño grupo cuya tarea es la de convertirse en factores de coordinación, síntesis e impulso

\section{BIBLIOGRAFÍA}

Allin, G. (1985). Spain's NATO Dilemma. Military Review, 65, pp. 61-73.

Alonso-Castrillo, S. (1996). La apuesta del centro: historia de la UCD. Madrid: Alianza.

Andrade Blanco, J. A. (2007). Del socialismo autogestionario a la OTAN: notas sobre el cambio ideológico en el PSOE durante la Transición a la democracia. Historia Actual Online, 14, pp. 97-106.

Bassols, R. (1995). España en Europa. Historia de la adhesión a la CE, 1957-85. Madrid: Política Exterior.

Calvo-Sotelo, L. (1978). Comisión de Asuntos Exteriores. Diario de Sesiones del Congreso de los Diputados, 44, pp. 1559-1588.

Calvo-Sotelo, L. (1982). Discursos e intervenciones públicas del Presidente del Gobierno Leopoldo Calvo-Sotelo. Madrid: Secretaría de Estado para la Información.

Calvo-Sotelo, L. (1990). Memoria viva de la Transición. Barcelona: Plaza y Janés/ Cambio 16

Calvo-Sotelo, L. (1999). Papeles de un cesante. La política desde la barrera. Barcelona: Galaxia Gutenberg.

Calvo-Sotelo, L. (2005). Sobre la Transición exterior. Madrid: Real Academia de Ciencias Morales y Políticas. de las diversas ramas de la Administración» (Nota informativa Relaciones con las CEE. Madrid, 26 de julio de 1978. ALCS, Relaciones con la CEE, caja 89, exp. 1).

2 Con el resultado de 126 votos a favor, 108 en contra y 2 abstenciones.

Calvo-Sotelo, L. (2010). Semblanza autobiográfica. En Calvo-Sotelo Ibáñez-Martín, P. (ed.). Leopoldo Calvo-Sotelo, un retrato intelectual. Madrid: Fundación Ortega-Marañón, Marcial Pons, pp. 27-30.

Calvo-Sotelo Ibáñez-Martín, P. (2010). Venturoso rincón, libros queridos. En CalvoSotelo Ibáñez-Martín, P. (ed.). Leopoldo Calvo-Sotelo, un retrato intelectual. Madrid: Fundación Ortega-Marañón, Marcial Pons, pp. 39-82.

Fernández Palomeque, P. (2010). Salvarse a sí mismo. La biblioteca de Leopoldo Calvo-Sotelo. En Calvo-Sotelo IbáñezMartín, P. (ed.), Leopoldo Calvo-Sotelo, un retrato intelectual. Madrid: Fundación Ortega-Marañón, Marcial Pons, pp. 83-96.

Fuentes, J. F. (2011). Adolfo Suárez. Biografía política. Barcelona: Planeta.

Gillespie, R. (1991). Historia del Partido Socialista Obrero Español. Madrid: Alianza.

Gómez Santos, M. (1982). Conversaciones con Leopoldo Calvo-Sotelo. Barcelona: Planeta.

Marquina, A. (1996). La política exterior de los Gobiernos de la Unión de Centro Democrático. En Tusell, J. y Soto, A. Historia de la Transición (1975-1986). Madrid: Alianza, pp. 182-215.
3 Por 186 votos a favor y 146 en contra.

4 Con el balance final de 106 votos a favor, 60 en contra y 1 abstención.

Martínez Lillo, P. (1996). Consenso y política exterior en la transición española. En Tusell, J. y Soto, A. Historia de la Transición (1975-1986). Madrid: Alianza, pp. 159-181.

Martínez Sánchez, J. A. (2011). El referéndum sobre la permanencia de España en la OTAN. UNISCI Discussion Papers, 26, pp. 283-310.

Míguez, A. (1989). El camino de la integración española en la OTAN. Proserpina. Revista de la Universidad Nacional de Educación a Distancia, 8, pp. 155-162.

Oreja Aguirre, M. (2011). Memoria y esperanza. Relatos de una vida. Madrid: La Esfera de los Libros.

Powell, Ch. (2010). Me he ido haciendo yo solo. En Calvo-Sotelo Ibáñez- Martín, P. (ed.). Leopoldo Calvo-Sotelo, un retrato intelectual. Madrid: Fundación OrtegaMarañón, Marcial Pons, pp. 99-111.

Powell, Ch. (2011). El amigo americano. España y Estados Unidos, de la dictadura a la democracia. Madrid: Galaxia Gutenberg/ Círculo de lectores.

Prego, V. (2000). Presidentes. Veinticinco años de historia narrada por los cuatro jefes de Gobierno de la democracia. Barcelona: Plaza y Janés. 